\title{
Sensor Node Deployment Strategy for Maintaining Wireless Sensor Network Communication Connectivity
}

\author{
Shigeaki TANABE, Kei SAWAI, Tsuyoshi SUZUKI \\ Tokyo Denki University, \\ School of Engineering, Department of Information and Communication Engineering, \\ Tokyo, Japan
}

\begin{abstract}
We propose a rescue robot sensor network system in which a teleoperated rescue robot sets up a wireless sensor network (WSN) to gather disaster information in post-disaster underground spaces. In this system, the rescue robot carries wireless sensor nodes (SNs) and deploys them between gateways in an underground space on demand by the operator's command to establish a safe approach path before rescue workers enter. However, a single communication path only is setup, because the rescue robot linearly deploys SNs between gateways. Hence, the rescue robot cannot be operated remotely if the communication path is disconnected by, for example, $\mathrm{SN}$ failure or changes in the environmental conditions. Therefore, SNs must be adaptively deployed so as to maintain WSN communication connectivity and negate such situations. This paper describes an SN deployment strategy for construction of a WSN robust to communication disconnection, caused by $\mathrm{SN}$ failure or deterioration of communications quality, in order to maintain communication connectivity between SNs. We thus propose an SN deployment strategy that uses redundant communication connection and ensures communication conditions between end-to-end communications of the WSN. The proposed strategy maintained communication conditions such that throughput between end-toend communications in the WSN. Experimental results verifying the efficacy of the proposed method are also described.
\end{abstract}

Keywords-wireless sensor network; deployment strategy; communication connectivity

\section{INTRODUCTION}

In recent years, disaster mitigation measures have been discussed in order to reduce the damage caused when a disaster occurs [1]. For disaster mitigation, various action plans have been devised and implemented in attempts to reduce damage and to facilitate early post-disaster rehabilitation and reconstruction. In particular, in an actual disaster zones, fire crews and rescue teams still must actually enter a post-disaster site to determine the current extent of the damage, because flexible responses are required according to the damage conditions. However, activities in such post-disaster situations present a high risk of personal injury to rescue workers due to secondary disasters, such as fire, or the collapse of walls and ceilings. Therefore, to reduce such risks, information gathering for early detection of possible threats is important in preventing secondary disasters, and actions to mitigate further disaster can be planned based on this information. Thus, continuous and exhaustive gathering and monitoring of information is necessary in the disaster zone in order to detect a secondary disaster rapidly, because secondary disasters can be caused by a number of factors that vary over time and locations.

Disaster information-gathering systems, for example, in the form of artificial satellites, unmanned aerial vehicles, and balloon flights are mainly used to gather disaster information over a wide area [2]. However, employment of these systems in

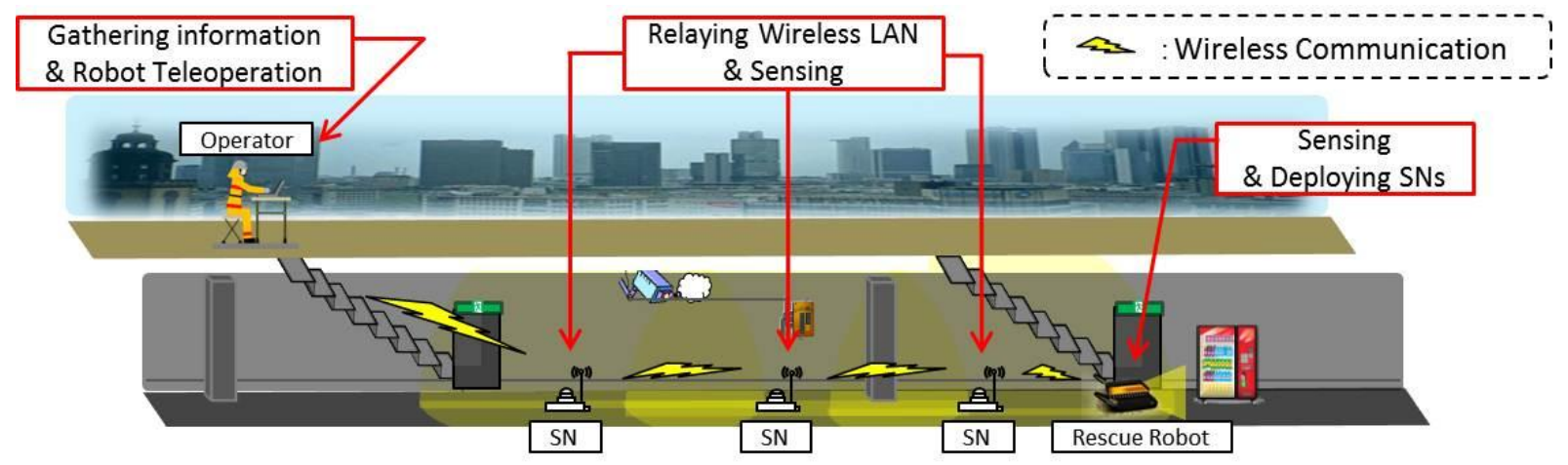

Figure 1. Conceptual sketch of information-gathering system in an underground space using RRSN 
underground spaces is difficult, and viable alternative methods for gathering information in such spaces have yet to be proposed. Moreover, many cases have been reported in which problems have actually been caused by existing disasterprevention equipment that has failed to operate during a disaster. Therefore, there is an increased need for informationgathering systems to determine the post-disaster status for disaster mitigation in underground spaces, such as subway stations and underground malls in urban areas. Underground spaces are particularly significant since, owing to their relative structural integrity, they are often expected to be used as evacuation shelters and as stores for emergency supplies.

Based on the above considerations, we have proposed a rescue robot sensor network (RRSN) system. In this system, a teleoperated mobile robot, such as a high-mobility rescue robot, sets up a wireless sensor network (WSN) to gather disaster information in post-disaster underground spaces (Fig. 1). WSNs consist of a number of small devices called sensor nodes (SNs), each of which is equipped with wireless communication functionality, various sensors, a processor, and a power source. A WSN is then a network system that can communicate and use data mutually gathered by the spatially distributed SNs. An ad hoc network that connects each SN one-by-one can be constructed by deploying a large number of SNs, and such a network is very easily enhanced compared with wired and fixed networks. WSNs can thus provide various services by collecting and processing the information acquired by the SNs, and WSNs technology is expected to be applicable in many fields, such as in cooperative monitoring of environmental conditions over large areas, in communication in sites where the construction of such infrastructure is difficult, and in information gathering for disaster-relief support.

Although establishing a wireless connection is generally difficult between ground level and an underground space, RRSN is able to create a communication link from a base station at ground level to a rescue mobile robot in an underground space via a wireless ad hoc network that connects each SN. Previously, we developed RRSN prototypes and verified their functionality experimentally [3]-[6]. In the proposed RRSN scenario [3], only a single communication path is setup because the rescue mobile robot linearly deploys SNs between the gateways in an underground space in order to establish a safe approach path for rescue workers. Hence, the rescue mobile robot cannot be remotely operated if a communication route is disconnected as a result of battery drain, SN failure, or changes in environmental factors, such as obstacles, radio interference, or radio wave conditions. Therefore, SNs must be adaptively deployed to maintain WSN communication connection in order to handle such unforeseeable circumstances. In addition, preserving the functionality of an information-gathering network is important, and maintenance is achieved by continually determining the state of the WSN and recovering the communication path if necessary. However, complex tasks for WSN restoration are not possible during the time-sensitive situation at a disaster site. Hence, to continue smooth teleoperation of rescue mobile robot under actual circumstances, an SN deployment method robust to disconnection of communication is vital. This paper thus proposes an SN deployment method for construction of a WSN robust to communication disconnection caused by $\mathrm{SN}$ failure or deterioration of communications quality. Experiments testing $\mathrm{SN}$ deployment and the maintenance of communications quality when using the proposed method are also described. These experiments verify the validity of the proposed.

The rest of the paper is organized as follows. Section 2 outlines works related to this study. Section 3 describes our system configuration and explains the proposed method of WSN construction. Section 4 evaluates the proposed method and presents the experimental results. Finally, concluding remarks are given in Section 5.

\section{RELATED WORKS}

Recently, many SN deployment strategies have been discussed in the WSN research field. In these strategies, deployment methods have been proposed based on evaluation scales that consider factors such as packet routing, energy efficiency, power-saving, and coverage area.

Chen et al. proposed an improved WSN routing protocol designed to maintain communication quality between SNs by referring only to the received signal strength indication (RSSI) value [7]. Iranli et al. have studied energy efficient strategies for deployment to construct a two-level WSN [8]. In that study, a control method for energy consumption in 2-hop networks is developed by utilizing an existing routing protocol. Zhang and Hou proposed a method for maintaining sensor coverage and communication connectivity by utilizing a WSN with a minimal number of SNs [9]. Furthermore, Zhang suggests a method of optimal geographical density control in large scale sensor networks that minimizes the number of SNs.

In the research into development of a mobile $\mathrm{SN}$ for construction of the WSN, Dantu et al. developed the Robomote, which added mobility to the Mica Mote. The Robomote can install WSN algorithms easily using the Mica Mote [10]. The MICAbot is a similar system to the Robomote, but it has higher mobility [11]. Suzuki et al. discussed WSN protocol-based research into a method where the mobile $\mathrm{SN}$ carries the data by physical movement when the WSN is disconnected [12][13].

Several SN deployment methods using mobile SNs and mobile robots to construct the WSN have been developed [14][16]. Parker et al. proposed a WSN construction method using an autonomous helicopter for environmental monitoring and urban search and rescue [17]. Umeki et al. proposed an ad-hoc network system, Sky Mesh, using a flying balloon for targeted disaster rescue support [18]. Also, deployment methods have been developed based on virtual interaction between the SNs based on several physical models, such as the potential field model and the fluid flow model [19][20].

However, none of these methods considered end-to-end network connectivity or network performance measures, such as throughput. Many researches are premised on communication link being maintained automatically. Moreover, evaluations in these methods are based solely on computer simulations. Hence, implementation of a practical wireless connection system was not attempted, and applying the methods is nontrivial for typical rescue mobile robot utilizing 2.4 GHz-band wireless communications. 


\section{SN DEPLOYMENT STRATEGY}

\section{A. SN deployment conditions}

To develop a test system for evaluating the proposed strategy, the conditions for SN deployment by RSSN are defined as follows.

Firstly, RRSN gathers environmental information in the vicinity of the gateways connecting the ground level and a first basement level, because this information is necessary for rescue workers to enter into the underground space. Under the Japanese Building Standards Law, safety stairs leading directly to ground level must be installed a maximum of every $30 \mathrm{~min}$ an underground space for escape during a disaster. As a result, our SNs are linearly deployed over a distance of about $40 \mathrm{~m}$, including the stairs and the passage between the gateways. Environmental information gathered by the RRSN is then continually transmitted to the operator, who can ascertain the disaster status within the underground space from this information.

The SN-loaded robot enters into the basement from the ground-side gateway through teleoperation by an operator at the base station. As the robot progresses it measures the communication conditions between the base station and its current position. When communication conditions satisfy the requirements specified by a deployment strategy, the robot indicates this information to the operator who decides an $\mathrm{SN}$ deployment point by referring to these conditions. The operator then gives a deployment command to the robot at the relevant location and the robot places an SN on the floor. The robot thus continues to measure the communication conditions between deployed SNs while moving toward a destination gateway, placing each SN in the same manner to setup the WSN.

Here, we assume that the extent of structural collapse is limited and that the environmental structure is largely maintained, because the underground space has earthquake resistance due to its structural integrity [21]. Therefore, the robot is not impeded in the space by unexpected obstacles resulting from the disaster. The robot and SNs are equipped with the wireless local area network (LAN) Institute of Electrical and Electronics Engineers (IEEE) standard, 802.11b. This standard is tolerant to communication disruption from obstacles and can connect to SNs within $50 \mathrm{~m}$ in 1-hop communication.

\section{B. Required specifications of communication}

The robot cannot be operated remotely via the WSN when the wireless communication quality has deteriorated between SNs. The wireless communication quality of the WSN constructed between the base station and the moving robot must always be maintained for smooth teleoperation of the robot. To satisfy this condition, a throughput of about $1.0 \mathrm{Mbps}$ is required for robot teleoperation to allow the passing of WSN data traffic [22]. However, for end-to-end multi-hop communication in a wireless ad hoc network, the throughput decreases as the number of SNs increases. For cases having greater than five hops, a throughput of $1.0 \mathrm{Mbps}$ is not ensured due to the upper limit of the IEEE $802.11 \mathrm{~b}$ standard being exceeded [23]. Thus, consideration of the number of SNs used in WSN communication is important for maintaining the necessary throughput. In the proposed RRSN, a WSN with up to 4-hop communication is constructed by the base station, SNs, and the robot (Fig. 2). The communication distance connected by these SNs (including the base station and robot) satisfies the above condition in terms of the IEEE $802.11 \mathrm{~b}$ specifications.

However, the throughput of end-to-end communication changes depending on the throughput between each SN. The distance between SNs cannot exceed a certain limit, since the throughput between two SN decreases as their distance increases. In contrast, when the distance between SNs is shortened, the WSN cannot cover the intended environment, because the number of SNs is restricted. In particular, the above communication condition is not satisfied when the SNs are deployed based on the nominal communication distance in wireless communication specifications, because the wireless communication performance dynamically changes depending on the environment in a post-disaster underground space. Therefore, determining the appropriate interval between SNs is important in order to cover our assumed environment while maintaining the required throughput. From the abovementioned requirements, we propose an $\mathrm{SN}$ deployment strategy that includes the following considerations:

- Determination of an appropriate SN interval.

- Setup of a WSN with a robust and fault-tolerant communication.

The following section describes an algorithm for determination of SN deployment positions that takes account of

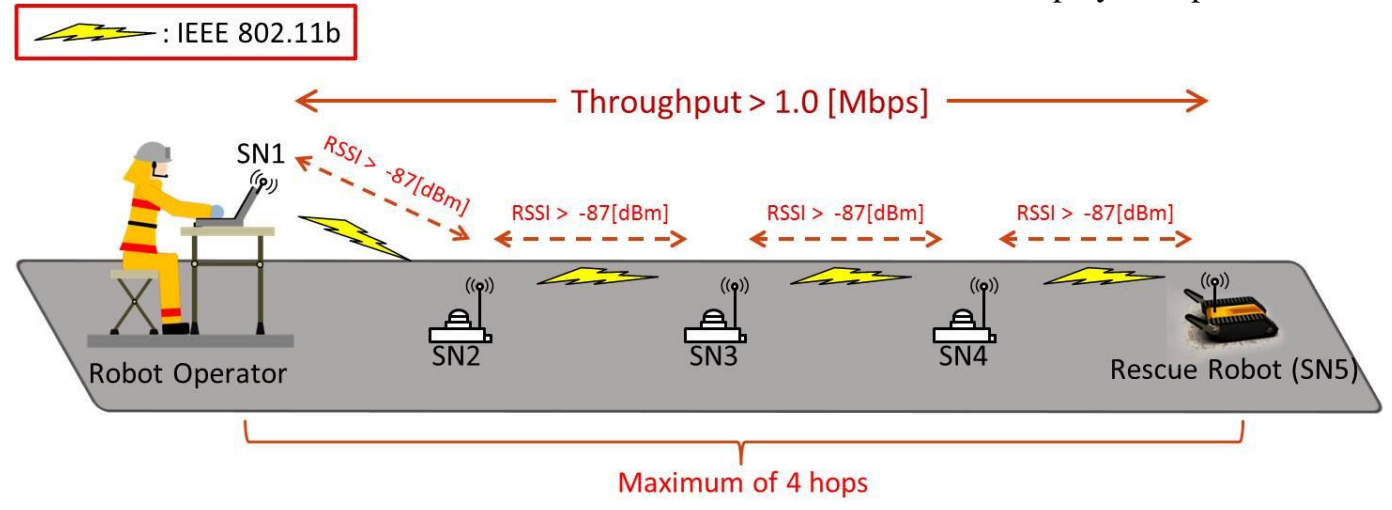

Figure 2. System configuration for SN deployment model that considers communication conditions 
the above condition of deploy SNs while maintaining a throughput of 1.0 Mbps.

\section{SN deployment model based on communication conditions}

To ensure communication performance between SNs, the robot measures throughput while transporting the SNs to their required locations. Thus, the robot confirms that the SNs are able to maintain a 1.0 Mbps throughput for end-to-end communication, and deploys SNs while ensuring the throughput between them. For 4-hop WSN communication, a 7.0 Mbps throughput must be maintained between each $\mathrm{SN}$ to guarantee a $1.0 \mathrm{Mbps}$ throughput for end-to-end communication. However, an auto-fallback system has been installed in the IEEE 802.11b standard that adjusts throughput automatically in response to changes of RSSI value. This system adjusts the upper limit of the throughput to less than 7.0 Mbps when the RSSI value falls below $-86 \mathrm{dBm}$. Therefore, both the throughput and RSSI values must be sustained to ensure the required communication performance, and the robot measures both the throughput and RSSI value between each SN accordingly. The robot moves continuously to the destination gateway while maintaining the $1.0 \mathrm{Mbps}$ of throughput required for end-to-end communication and an RSSI value of $86 \mathrm{dBm}$ between each SN. When the RSSI value starts to decay, the robot places the SN before the RSSI values fall below $-86 \mathrm{dBm}$ (Fig. 2). The robot thus sets up the WSN by repeatedly placing SNs on the floor, one by one, by measuring the throughput and RSSI value between SNs. This method is expected to provide SN deployment and smooth robot teleoperation adaptive to changes in communication performance caused by environmental interference.

\section{SN deployment model based on redundant communication connection}

Because the disconnection of communication caused by $\mathrm{SN}$ failure is a severe problem in setting up a WSN, several research studies have attempted to improve the fault tolerant performance of WSNs [24][25]. In conventional methods, a large number of SNs are deployed for WSN construction, and the communication path then changes according to $\mathrm{SN}$ failure. In our system, however, these methods cannot be used owing to our restricted number of SNs and linear deployment design. Thus, we instead propose a redundant placement method for SNs. That is, two SNs are placed within an area where the RSSI value from last $\mathrm{SN}$ is greater than $-86 \mathrm{dBm}$ and throughput is greater than 7.0 Mbps. Upon failure, automatic restoration of the communication path to the robot is then possible by connecting to the next running $\mathrm{SN}$, and the 1.0 Mbps of throughput between the end-to-end communications in the WSN is maintained if the network routing is changed (Fig. $3)$.

\section{E. SN deployment strategy based on both models}

We lastly propose an SN deployment strategy that uses redundant communication connection and ensures communication conditions between end-to-end communications of the WSN. In this section, an SN deployment algorithm using the robot based on abovementioned models is described.

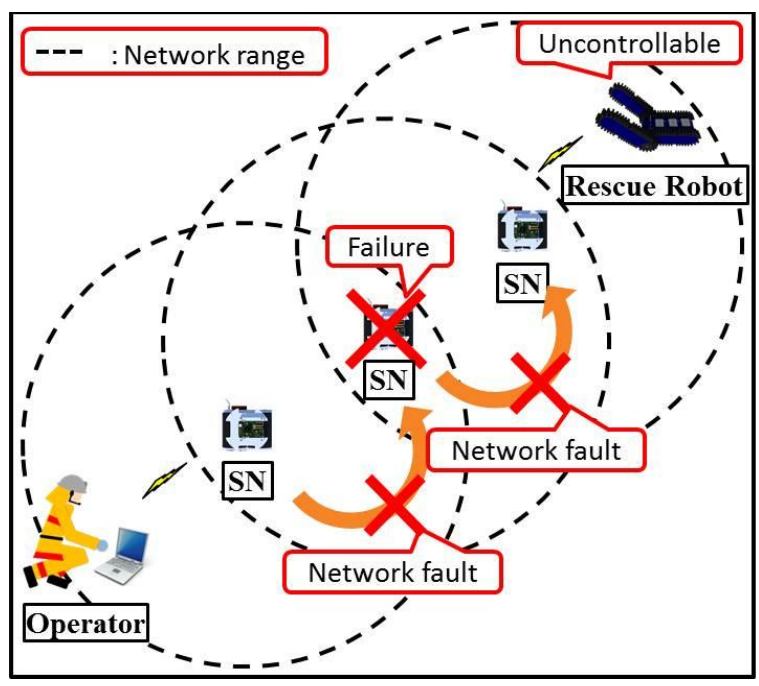

(a) SN deployment without considering redundant communication connection

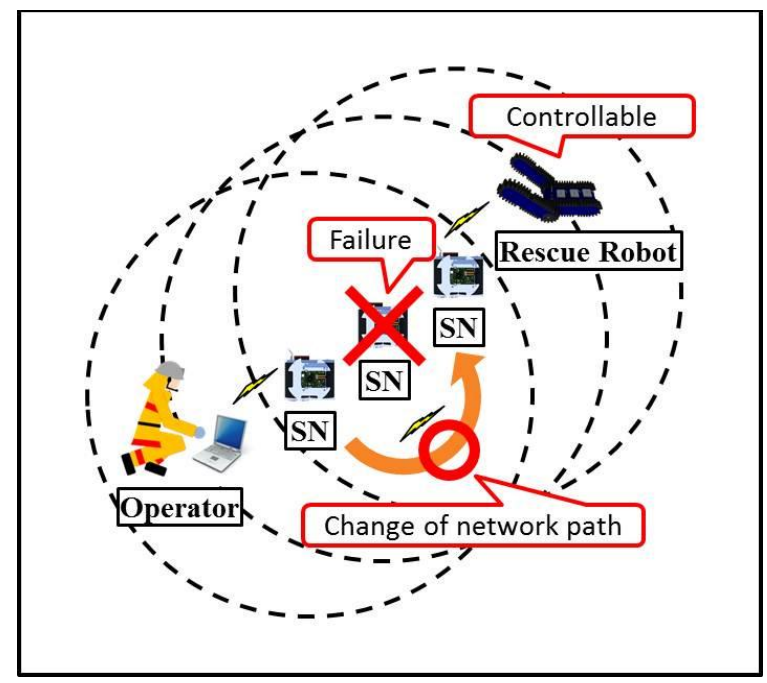

(b) $\mathrm{SN}$ deployment with considering redundant communication connection

Figure 3. SN deployment model considering redundant communication connection

Figure 4 shows the steps of the algorithm.

i) As shown by path (1) in Fig. 4, the SN-loaded robot moves from base station to a destination gateway by teleoperation. While moving, the robot measures both the RSSI value between the base station and current position, and the throughput between end-to-end communications.

ii) When the throughput or RSSI value starts to degrade, the robot stops before they reach their relevant thresholds (1.0 Mbps and $-86 \mathrm{dBm}$, respectively). The robot measures distance it has moved from the start point (P2 in Fig.4) and stores this stop position.

iii) The robot transmits the communication conditions data to the operator. The operator confirms the information and sends an SN deployment command.

iv) The robot moves back half of the distance it has traveled (path (2)), and places first SN (SN2) at point P1. 


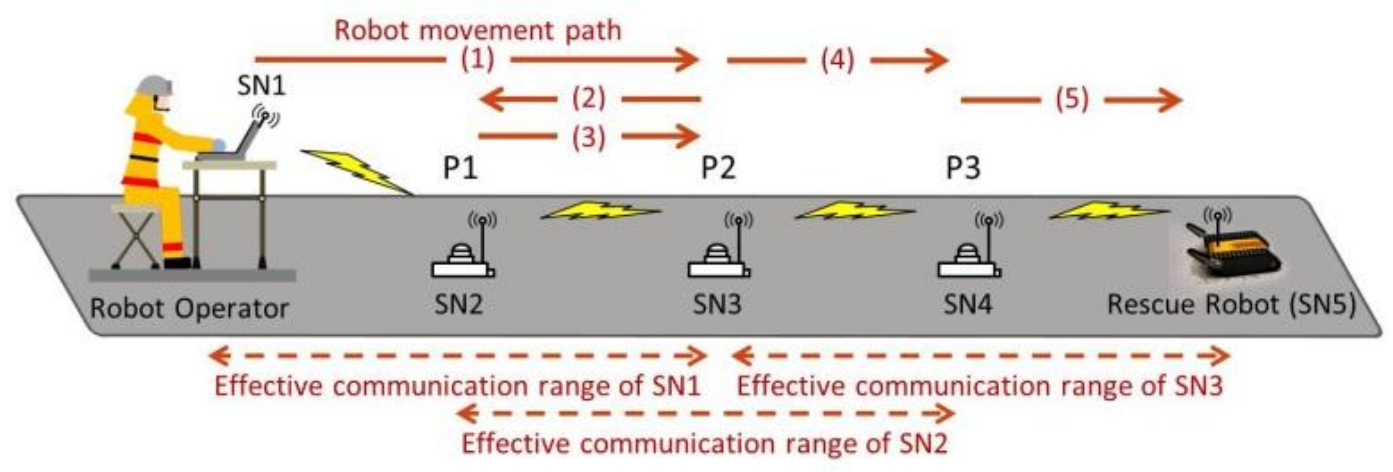

Figure 4. Steps in WSN deployment algorithm

v) After that, the robot moves forward to the stored stopping point P2 and places SN3, and again measures the throughput between the base station and current position to confirm the end-to-end communication conditions.

vi) In a similar manner, the robot continues to move forward (path (4)), measuring the communication conditions between SN2 and its current position. The robot stops before the communication performance degrades below the thresholds and places SN4 at P3.

vii)The robot continues moving in this way (path (5)) until it has arrived at the destination gateway.

\section{Evaluation of SN DePloyment StRategy}

\section{A. Experiment for confirming SN communication conditions}

We evaluated our proposed development strategy in a passageway of $40 \mathrm{~m}$ in length. In this evaluation, we measured the throughput to determine the communication quality between the base $\mathrm{SN}$ and a destination $\mathrm{SN}$.

The SN deployment interval was decided by RSSI; that is, the electrical field density between SNs. The threshold level for maintaining communication quality was defined as $-86 \mathrm{dBm}$, and the extended distance of the WSN was calculated based on sum of the SN intervals. The RSSI was measured every $5 \mathrm{~m}$ that the robot moved.

Table I shows the SN specifications. Armadillo-300 (AtMark Techno Ltd.) was used as the SN controller. For RSSI measurements, we used the "wlanconfig" command contained in the Wireless Tools package in the Debian Linux distribution. To measure the throughput of a packet, "utest" (NTT Communications Ltd.) was used.

The crawler-type mobile robot (TOPY industry Ltd.) in Fig. 5 was adopted for use as the rescue mobile robot in this experiment.

24 bytes data containing operation modes and each actuator velocity were transmitted as the robot operation commands. The communication status measurement commands described above were also transmitted with parameters. 67 bytes data containing each actuator velocity, current values, a tilt angle of the robot and a range sensor data were received as the robot status. The RSSI value and the throughput were also received as the current communication conditions.
Image data was not used in this evaluation, but when the throughput of $1.0 \mathrm{Mbps}$ is maintained between end-to-end communications, it can be communicated enough.

Figure 6 shows the experimental results. The maximum extended distance of the WSN was $95 \mathrm{~m}$ under the conditions of 4-hop communication, and RSSI and throughput values exceeding $-86 \mathrm{dBm}$ and 1.0 Mbps, respectively. Therefore, we established that the proposed strategy could be applied in our assumed environment of $40 \mathrm{~m}$.

TABLE I. SN SPECIFICATIONS

\begin{tabular}{|l|l|}
\hline \multicolumn{2}{|c|}{ Devices and tools } \\
\hline Linux Controller & Armadillo-300 \\
\hline Wireless LAN & IEEE $802.11 \mathrm{~b}(2.4 \mathrm{GHz})$ \\
\hline Measurement Software & Wireless tools and utest \\
\hline
\end{tabular}

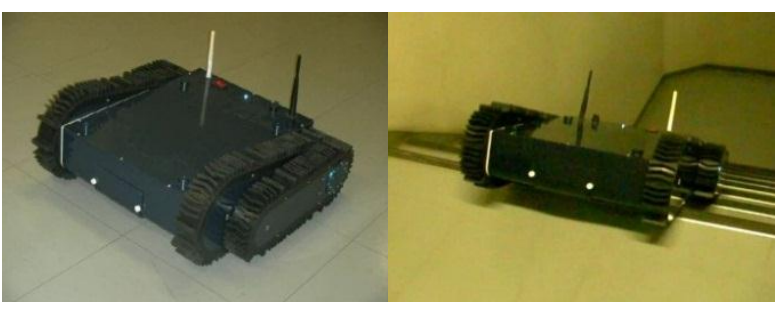

Figure 5. Crawler-type mobile robot

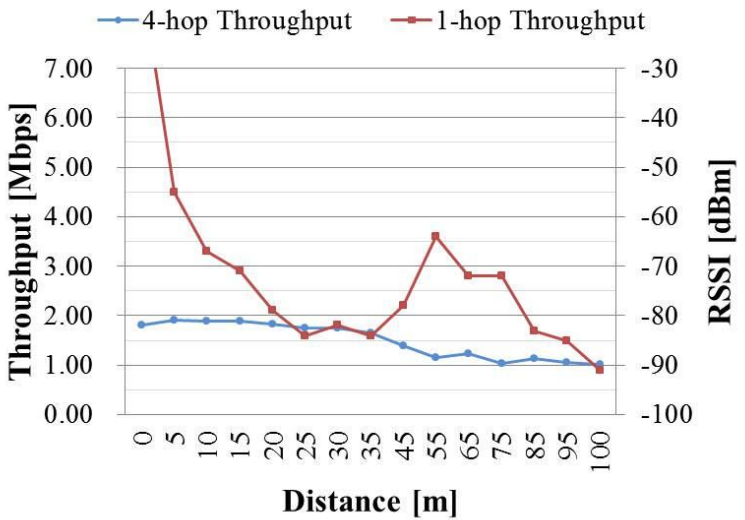

Figure 6. Example measurement results for throughput and RSSI 


\section{B. Evaluation of SN deployment strategy}

We measured the WSN communication conditions in order to evaluate the fault-tolerant capability of the proposed strategy. For this evaluation, we compared two deployment strategies. Strategy 1 determined SN placement based on the maximum range described in the communication specifications. Strategy 2 was the proposed strategy, where SN positions were determined by referring the maximum extended distance shown in Fig. 6. For WSN construction using the proposed strategy, by referring Fig. 6 , the $\mathrm{SN}$ placement positions were calculated as $0,35,65,85$, and $95 \mathrm{~m}$. The power supply of one $\mathrm{SN}$ was then randomly switched off to simulate SN failure. The experimental environment was same as in the previous experiment.

When SN failure occurred, the communication path changed in both strategies, and the throughput and RSSI values between the SNs in new communication path were measured. Tables II and III shows the results when SN2, SN3, and SN4 fail while using Strategies 1 and 2, respectively. For Strategy 1 the communication conditions decayed to $-87 \mathrm{dBm}$ or less and 1.0 Mbps or less. However, for Strategy 2, communication conditions were maintained above $-87 \mathrm{dBm}$ and $1.0 \mathrm{Mbps}$. Therefore, both communication maintenance and failure resistance in using the proposed strategy are verified.

Although the proposed strategy maintains communication connectivity against the communication disconnection caused by SN failure, measurement results of the communication conditions change depending on the environment condition.

The communication link of the RRSN is disconnected if the communication conditions are degraded dramatically. For such case, the other strategy must be applied to solve communication disconnection. For example, we have proposed WSN reconstruction method that the robots deploy additional or alternate SNs to necessary sites to restore communication links [3]. When a wireless communication cannot be available, the strategy to gather as much information as possible using wired mobile robot is also considered.

TABLE II. EXPERIMENTAL RESULT USING THE MAXIMUM EXTENDED WSN BASED ON COMMUNICATION DISTANCE SPECIFICATIONS (STRATEGY 1)

\begin{tabular}{|c|c|c|}
\hline Fault SN & RSSI [dBm] & End-to-End Throughput [Mbps] \\
\hline SN2 & -89 & Immeasurable level \\
\hline SN3 & -87 & 0.267 \\
\hline SN4 & -91 & 0.507 \\
\hline
\end{tabular}

TABLE III. EXPERIMENTAL RESULTS USING PROPOSED DEPLOYMENT STRATEGY (STRATEGY 2)

\begin{tabular}{|c|c|c|}
\hline Fault $\boldsymbol{S N}$ & $\boldsymbol{R S S I}[\mathbf{d B m}]$ & End-to-End Throughput [Mbps] \\
\hline SN2 & -79 & 1.132 \\
\hline SN3 & -86 & 1.041 \\
\hline SN4 & -81 & 1.377 \\
\hline
\end{tabular}

\section{DisCUSSION AND FUTURE DIRECTION}

This paper has focused on a SN deployment strategy considering the communication conditions and redundancy. In this paper, the key result is that SN deployment positions can be determined adaptively according to the communication conditions, and communication connectivity can be maintained against the communication disconnection caused by SN failure. However, it is difficult to apply this prototype system to actual post-disaster situations. Therefore, we are now developing new crawler robots for SN deployment and information gathering in post-disaster situations based on the findings obtained from our studies. The proposed strategy in this paper will be applied to new robots. These component technologies are integrated, and the performance of the RRSN must be further developed and improved to enable it to be applied practically in assumed environment to gather information on the effects of disasters in underground spaces.

\section{CONCLUSION}

This paper has proposed a WSN deployment strategy that maintains communication conditions and has a fault-tolerant communication connection. The proposed strategy maintained communication conditions such that throughput between endto-end communications in the WSN enables smooth teleoperation of a mobile robot in a post-disaster underground space. Experimental results showed the effectiveness of the proposed strategy.

This strategy, which prevents communication disconnection caused by SN failure, is considered effective for WSN deployment in actual disaster scenarios, because the rapid implementation of actions to mitigate secondary disasters in disaster zones requires the stable referral of disaster information. We will apply the proposed strategy to WSN deployment in practical underground spaces in the future.

\section{ACKNOWLEDGMENT}

This work was partially supported by the Research Institute for Science and Technology of Tokyo Denki University, Grant Number Za10-01 / Japan.

\section{REFERENCES}

[1] Y. Kawata, "The great Hanshin-Awaji earthquake disaster: damage, social response, and recovery," Journal of Natural Disaster Science, Vol. 17, No. 2, pp.1-12, 1995.

[2] H. Kawakata, Y. Kawata, H. Hayashi, T. Tanaka, K. C. Topping, K. Yamori, P. Yoshitomi, G. Urakawa and T. Kugai, "Building an integrated database management system of information on disaster hazard, risk, and recovery process" Annuals of Disas. Prev. Res. Inst., Kyoto Univ., No.47 C, 2004.

[3] T. Suzuki, R. Sugizaki, K. Kawabata Y. Hada and Y. Tobe, "Autonomous deployment and restoration of sensor network using mobile robots,"International Journal of Advanced Robotic Systems, ISSN 1729-8806, Vol. 7, No. 2, June 2010, pp.105-114

[4] H. Sato, K. Kawabata, T. Yugo, H. Kaetsu and T. Suzuki, "Wireless camera nodes deployment by a teleoperated mobile robot for construction of sensor network," ICROS-SICE International Joint Conference 2009 (ICCAS-SICE2009), pp.3726-3730, August 18-21, Fukuoka International Congress Center, Fukuoka, JAPAN, 2009 
[5] K.Sawai, T.Suzuki, H.Kono, Y.Hada and K.Kawabata, "Development of a sensor node with impact-resistance capability for gathering disaster area information," 2008 International Symposium on Nonlinear Theory and its Applications (NOLTA2008), pp.17-20 (A1L-C2), ISBN: 978-488552-234-5, Budapest, Hungary, September 7-10, 2008.

[6] H. Sato, K. Kawabata and T. Suzuki, "Information Gathering by wireless camera node with Passive Pendulum Mechanism," International Conference on Control, Automation and Systems 2008 (ICCAS2008), pp.137-140, October 14-17, Seoul, Korea, 2008.

[7] F. Chen, C. Wu, P. Ji and Y. Zhang, "A communication quality improved routing protocol for wireless sensor network", Automation and Logistics, 2009. ICAL '09. IEEE International Conference on Digital Object Identifier, pp. $616-620,2009$.

[8] A. Iranli, M. Maleki, M. Pedram, "Energy efficient strategies for deployment of a two-level wireless sensor network," Proceedings of the 2005 International Symposium on Low Power Electronics and Design 2005 (ISLPED '05), pp. 233-238, Southern California Univ., Los Angeles, CA, USA, August 8-10, 2005.

[9] H. Zhang, J. Hou, "Maintaining sensing coverage and connectivity in large sensor networks", Ad Hoc and Sensor Wireless Networks, Vol. 1, No. 1-2. 2005.

[10] K. Dantu, M. Rahimi, H. Shah, S. Babel, A. Dhariwal. and S. S. Gaurav., "Robomote: enabling mobility in sensor networks," Fourth International Symposium on Information Processing in Sensor Networks (IPSN2005), pp. 404-409, 2005.

[11] M. B. McMickell, B. Goodwine and L. A. Montestruque, "MICAbot: A robotic platform for large-scale distributed robotics," Proceedings of the IEEE International Conference on Robotics and Automation (ICRA2003), 2, 1600-1605, 2003.

[12] R. Suzuki, K. Makimura, H. Saito and Y. Tobe, "Prototype of a sensor network with moving nodes, "Special Issue on INSS 2004, Transaction SICE, E-S-1, pp. 52-57, 2006.

[13] R. Suzuki, K. Sezaki and Y. Tobe, "A Protocol for policy-based session control in disruption tolerant sensor networks. Special section on Ubiquitous Sensor Networks, IEICE TRANSACTIONS on Communications, E90-B(12), pp. 3426-3433, 2007.

[14] M. A. Batalin, and G. Sukhatme, "Sensor coverage using mobile robots and stationary nodes," Proceedings of the SPIE (SPIE2002), No. 4868, pp. 269-276, 2002.

[15] S. Miyama, M. Imai and Y. Anzai, "Rescue robot under disaster situation: position acquisition with omni-directional sensor," Proceedings of 2003 IEEE/RSJ International Conference on Intelligent Robots and Systems (IROS2003), pp. 3132-3137, 2003.

[16] M. Sugano, T. Kawazoe, Y. Ohta and M. Murata, "An indoor localization system using RSSI measurement of a wireless sensor network based on the ZigBee standard," Proceedings of the sixth IASTED International Multi-Conference on Wireless and Optical Communication, pp. 504-508, 2006.
[17] L. E. Parker, B. Kannan, F. Xiaoquan and T. Yifan, "Heterogeneous mobile sensor net deployment using robot herding and line of sight formations," Proceedings of 2003 IEEE/RSJ International Conference on Intelligent Robots and Systems (IROS2003), Vol. 3, pp. 2488-2493, 2003.

[18] T. Umeki, H. Okada and K. Mase, "Evaluation of Wireless channel quality for an ad hoc network in the sky, SKYMESH," The Sixth International Symposium on Wireless Communication Systems 2009 (ISWCS'09), pp.585-589, 2009.

[19] A. Howard, M. J. Matric and G. S. Sukhatme, "Mobile sensor network deployment using potential fields: A distributed, scalable solution to the area coverage problem," Distributed Autonomous Robotics Systems 5, Springer-Verlag, pp. 299-308, 2002.

[20] M. R. Pac, A. M. Erkmen and I. Erkmen, "Scalable self-deployment of mobile sensor networks: A fluid dynamics approach," Proceedings of 2006 IEEE/RSJ International Conference on Intelligent Robots and Systems (IROS2006), pp. 1446-1451, 2006.

[21] P. J. Godard, "Urban Underground Space and Benefits of Going Underground", Proceedings of World Tunnel Congress 2004 and 30th ITA General Assembly, pp.1-9, Singapore, pp. 22-27, May 2004.

[22] J. Yamashita, K. Sawai, Y. Kimitsuka, T. Suzuki,Y. Tobe, "The design of direct deployment method of sensor nodes by utilizing a rescue robots in disaster areas," SICE Annual Conference 2008, pp183, 2B3-4, 6 Dec. 2008 (in Japanese)

[23] K. SAWAI, T. SUZUKI, "Evaluation of network construction method considering reduce of throughput by utilizing rescue robot," Proceedings of the 2010 JSME Conference on Robotics and Mechatronics, No. 10-4, 1A2-C09(2), 2010 (in Japanese)

[24] M. Ishizuka, M. Aida, "Stochastic Node Placement Improving Fault Tolerance in Wireless Sensor Networks," Institute of Electronics, Information, and Communication Engineers, J88-B(11), pp. 2181-2191, 2005.

[25] B. Krishnamachari, S. Iyengar, "Distributed Bayesian algorithms for fault-tolerant event region detection in wireless sensor networks," IEEE TRANSACTIONS ON COMPUTERS, Vol. 53, No. 3, pp. 241-249, March 2004.

\section{AUTHORS' PROFILE}

Shigeaki TANABE - Graduate school student at Tokyo Denki University. His research interests include sensor network communication and robotassisted sensor networks.

Kei SAWAI - Assistant professor at Tokyo Denki University. His research interests include networked robotics and robot-assisted sensor networks.

Tsuyoshi SUZUKI - Professor at Tokyo Denki University. His research interests include multi-robot systems, human-robot interaction, telerobotic systems, networked robotics, and robot-assisted sensor networks. 